\title{
Time-Resolved Photodissociation of Singly Protonated Peptides with an Arginine at the N-Terminus: A Statistical Interpretation
}

\author{
So Hee Yoon, Yeon Ji Chung, and Myung Soo Kim \\ Department of Chemistry, Seoul National University, Seoul, Korea
}

\begin{abstract}
Time-evolution of product ion signals in ultraviolet photodissociation (UV-PD) of singly protonated peptides with an arginine at the $\mathrm{N}$-terminus was investigated by using a tandem time-of-flight mass spectrometer equipped with a cell floated at high voltage. Observation of different time-evolution patterns for different product ion types-an apparently nonstatistical behavior-could be explained within the statistical framework by invoking consecutive formation of some product ions and broad internal energy distributions for precursor ions. $a_{n}+1$ and $b_{n}$ ions were taken as the primary product ions from this type of peptide ions. Spectral characteristics in post-source decay, UV-PD, and collisionally activated dissociation at low and high kinetic energies could be explained via rough statistical calculation of rate constants. Specifically, the striking characteristics in high-energy CAD and UV-PD-dominance of $a_{n}$ and $d_{n}$ formed via $a_{n}+1$-were not due to the peculiarity of the excitation processes themselves, but due to quenching of the $b_{n}$ channels caused by the presence of arginine. (J Am Soc Mass Spectrom 2008, 19, 645-655) (c) 2008 American Society for Mass Spectrometry
\end{abstract}

$\mathrm{T}$ Tandem mass spectrometry has been widely used for identification and sequence determination of polypeptides and proteins [1-3]. In spite of extensive studies made so far, fundamental understanding on the dissociation of activated peptide ions observed by tandem mass spectrometry is still lacking. This is in contrast with the case of the dissociation of small polyatomic ions, for which nearly quantitative theoretical description and even prediction are possible [4]. The main reasons for such a lack of fundamental understanding are experimental and computational difficulties to get structural, mechanistic, and kinetic data for large polyatomic systems consisting of more than 100 atoms. In this regard, it is to be mentioned that we recently developed a systematic method to calculate sequence-specific statistical (Rice-Ramsperger-KasselMarcus, RRKM) rate constants for dissociations of peptide and protein ions [5-7].

The most popular method to activate a peptide ion and hence to induce its dissociation is the collisionally activated dissociation (CAD) [8-12]. The kinetic energy of a peptide ion is an important factor affecting the CAD spectral pattern. Hence, CAD of a peptide ion is classified into two categories, low (around $100 \mathrm{eV}$ ) and high (higher than $1 \mathrm{keV}$ ) energy regimes. In the lowenergy CAD [11], which is typically done with triple quadrupole ion trap and ion cyclotron resonance mass spectrometers, a peptide ion gains sufficient energy for

Address reprint requests to Dr. M. S. Kim, Department of Chemistry, Seoul National University, Seoul 151-742, Korea. E-mail: myungsoo@snu.ac.kr dissociation usually via multiple collisions, each collision supplying rather small amounts of internal energy through vibrational excitation. Most of the product ions are $b$ and $y$ types (see Scheme 1) formed via rearrangement reactions.

Presence of arginine, proline and aspartic acid residues and the charge state of a peptide ion are known to affect the relative intensities of these product ions. The "mobile proton" model $[13,14]$ has been devised to explain this. In the high-energy CAD [10], which is usually done with sector and time-of-flight (TOF) mass spectrometers, a peptide ion gains more internal energy than the above through electronic excitation. In the case of singly protonated peptides without an arginine residue, most of the product ions are $b$ and $y$ types, and some $a$ type also, just as in low-energy CAD. When an arginine residue is present, a rather dramatic spectral change occurs; $a$ and $d$ type product ions dominate with an arginine residue at the $\mathrm{N}$-terminus while $x, y, v$, and $w$ types dominate with the same residue at the Cterminus. For the former case, which is related to the main subject of this paper, Biemann and coworkers [15] suggested that $a$ and $d$ type products were formed via radical site reactions of $a+1$ type ions generated via simple bond cleavage of the precursor ion.

Photoexcitation is another useful method to activate a peptide ion. Infrared multiphoton dissociation (IRMPD) [16, 17] is widely practiced, especially in trapping type instruments. In IRMPD, internal energy is supplied via sequential multiple vibrational excitations, as in low-energy CAD. Hence, the overall spectral 
<smiles></smiles><smiles>[R]C=N[Al]C(=O)C([R])N</smiles>

$a_{n}$

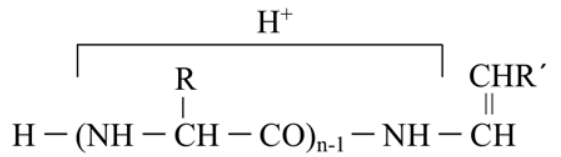

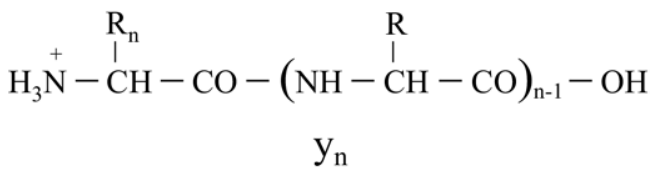

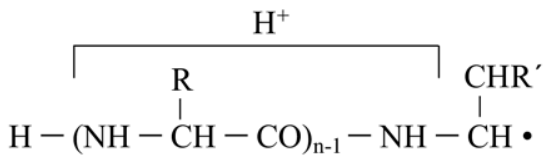

$\mathrm{a}_{\mathrm{n}}+1$

$\mathrm{d}_{\mathrm{n}}$

Scheme 1. Structures of the product ions of interest in this work.

feature in IRMPD is rather similar to that in low-energy CAD. Even though photodissociation (PD) by ultraviolet (UV) or vacuum ultraviolet (VUV) light, or UV-PD, has been widely used to study the structure and dissociation dynamics of small polyatomic ions [18, 19], decent tandem mass spectra of peptide ions measured by UV-PD have been reported only recently [20-24]. Ion activation in this technique occurs via electronic excitation, as in high-energy CAD. The fact that the excited electronic state accessed can be controlled by choosing the wavelength is one of the advantages of UV-PD. Tandem mass spectra of singly protonated peptides measured by this technique have been found to be quite similar to those by high-energy CAD [25].

Recently, we reported a new technique [26] to get temporal information on PD of protonated peptides generated by matrix-assisted laser desorption ionization (MALDI). A peptide ion $\left(\mathrm{MH}^{+}\right)$beam was irradiated by $266 \mathrm{~nm}$ PD laser beam inside a cell floated at high voltage. Since the final kinetic energies of the same product ions $\left(m_{1}^{+}\right)$formed inside (in-cell) and outside (post-cell) the cell differed, the two could be separated by a reflectron analyzer. Also, additional peaks appeared between the two in the TOF spectra. These were due to consecutive formation $\left(\mathrm{MH}^{+} \rightarrow m_{i}^{+} \rightarrow m_{1}^{+}\right)$of the product ion via intermediate ions $\left(m_{i}^{+}\right)$with the first step occurring inside the cell and the second step outside. A method based on SIMION calculation was developed to estimate the intermediate ion masses. Identification of major consecutive dissociation channels for model peptide ions resulted in fragmentation maps for the photoexcited peptide ions.

Since the main purposes of the previous work were to develop the new technique and to get a spectral overview, peptide ions with an arginine residue-there is some controversy on their dissociation mechanism $[21,23,27]$-were intentionally excluded in the study. With the working principle of the technique well established, we attempted to obtain and analyze the timeresolved PD data for peptide ions with an arginine residue at the $\mathrm{N}$-terminus. Some of the observations, such as different time-evolution patterns for different reactions, were apparently in contradiction with the statistical picture of unimolecular reaction. It has been found, however, that an explanation of the observations within the statistical framework is possible by taking into account the internal energy distributions for peptide ions generated by MALDI and occurrence of consecutive reactions. The results are reported in this paper.

\section{Experimental}

Details of the homebuilt tandem TOF instrument and its operation were reported previously [26]. A brief description is as follows. The instrument consists of a MALDI source with delayed extraction, a first-stage TOF analyzer to time-separate the prompt ions generated by MALDI, an ion gate, a PD cell, and a secondstage TOF analyzer equipped with a reflectron. The ion gate is located immediately in front of the first time focus and the PD cell at the first time focus. A deflection system [28] to eliminate fragment ions generated by post-source decay (PSD) is installed between the ion source and the ion gate; $337 \mathrm{~nm}$ output from a nitrogen laser (MNL205-C; Lasertechnik Berlin, Berlin, Germany) is used for MALDI; $20 \mathrm{kV}$ DC and $1.8 \mathrm{kV} \mathrm{AC}$ pulse are used in the ion source for delayed extraction/ acceleration. The potential inside the reflectron has linear and quadratic components. The final electrode of 


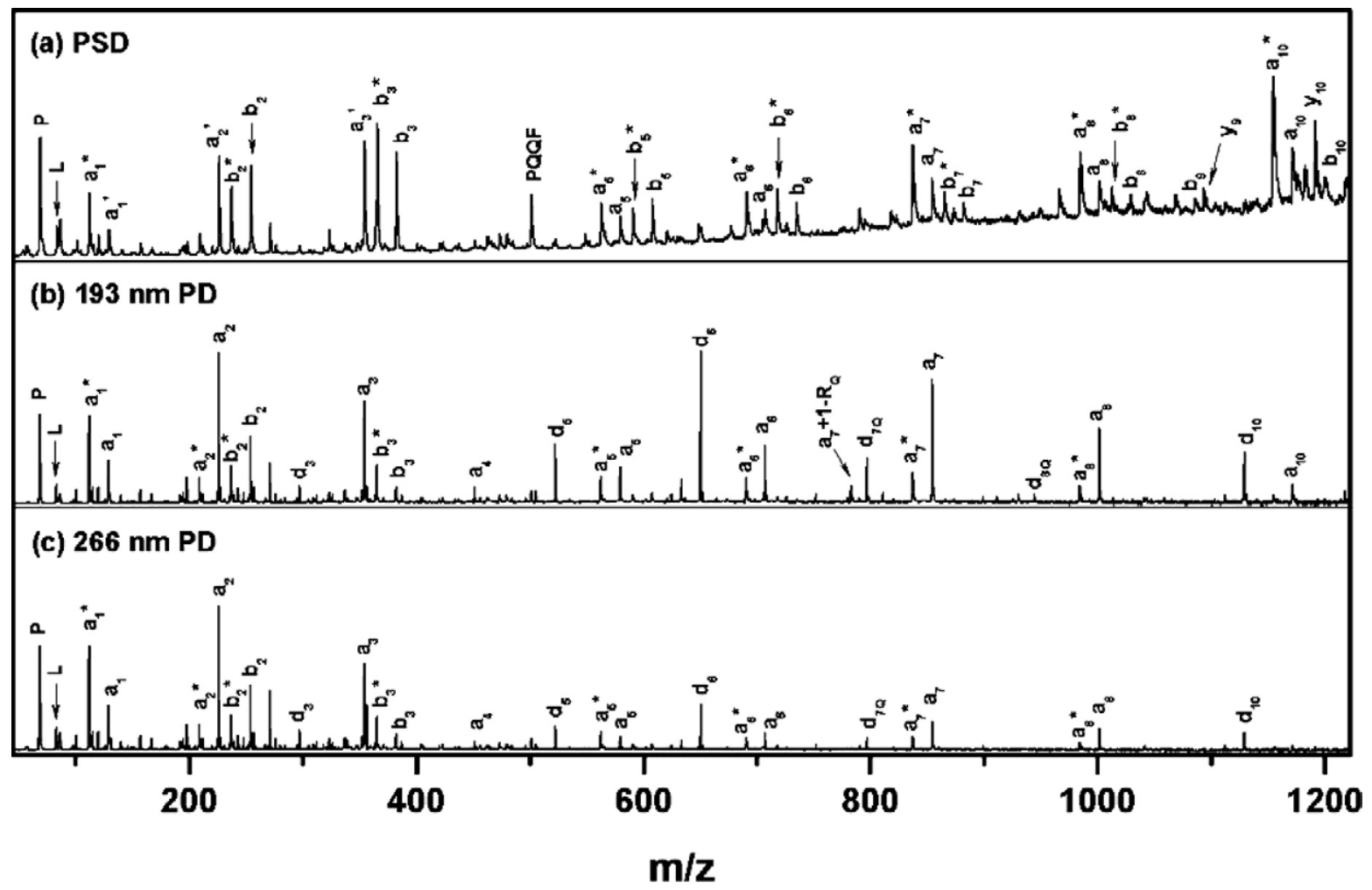

Figure 1. (a) PSD, (b) $193 \mathrm{~nm}$ PD, and (c) $266 \mathrm{~nm}$ PD spectra of singly protonated substance P. Laser-off spectrum was subtracted from laser-on spectrum to obtain each PD spectrum. $a_{n}{ }^{*}$ and $b_{n}{ }^{*}$ indicate $a_{n}-\mathrm{NH}_{3}$ and $b_{n}-\mathrm{NH}_{3}$, respectively.

the reflectron is kept at $25 \mathrm{kV}$ in normal operation. A pulsed PD laser, either $266 \mathrm{~nm}$ output from a Nd:YAG laser (Surelite III-10; Continuum, Santa Clara, CA) or $193 \mathrm{~nm}$ output from an ArF excimer laser (PSX-100; MPB Communication Inc., Montreal, Quebec, Canada), is irradiated at the center of the PD cell perpendicularly to the ion beam direction. Two delay generators control timing for MALDI laser irradiation, AC pulsing for delayed extraction, ion gate opening, and PD laser pulsing. The PD laser pulse is synchronized with the A peak (the lowest-mass isotopomer) of the precursor ion selected for PD. PD laser intensity is set such that intensity-dependent spectral change is insignificant. The PSD spectrum under the same experimental condition is recorded without the PD laser pulse. The output from the MCP detector is digitized by an A/D card and treated to minimize electrical noise [29]. Finally, the laser-off spectrum (PSD) is subtracted from the laser-on spectrum to obtain the laser-induced change or the PD spectrum.

RLLAPITAY and RVYIPF with better than $90 \%$ purity were purchased from Peptron (Daejon, Korea). Substance P (RPKPQQFFGLM- $\mathrm{NH}_{2}$ ) with better than 98\% purity, the matrix ( $\alpha$-cyano-4-hydroxycinammic acid), and other chemicals were purchased from Sigma (St. Louis, MO). A matrix solution prepared daily using acetonitrile and $0.1 \%$ trifluoroacetic acid was mixed with a peptide solution. The final peptide concentration prepared for PD experiment was $10 \mathrm{pmol} / \mu \mathrm{L} ; 1 \mu \mathrm{L}$ of the solution was loaded on the sample plate. The method to prepare a deuterated MALDI sample is the same as above except that $\mathrm{D}_{2} \mathrm{O}$ (better than $99.96 \%$ isotopic purity) was used as the solvent. A sample plate was placed inside the sample introduction system, which was flushed with $\mathrm{D}_{2} \mathrm{O}$, evacuated by a rotary pump, and then filled with dry nitrogen. Peptide/matrix mixture was loaded on the plate through a septum by syringe. The system was evacuated after $1 \mathrm{~min}$ and the sample plate was introduced to the ion source.

\section{Results and Discussion}

We carried out time-resolved study for several singly protonated peptides with an arginine at the N-terminus. Their characteristics were very similar. Details will be explained here using the singly protonated substance $\mathrm{P}$ as an example because rather extensive studies on the fragmentation of this ion in high-energy CAD [15] and in PSD [30] were reported previously. The PSD spectrum of the singly protonated substance $P$ and the PD spectra recorded at 193 and $266 \mathrm{~nm}$ are shown in Figure 1. The PSD spectrum looks similar to the one reported by Spengler et al. [30]. Peaks in the PD spectra look sharper than those in PSD. This is because mono isotopomeric selection has been made in PD via synchronization of the laser pulse with the A peak pulse of the ion beam. Even though the intensity patterns in the two PD spectra are somewhat different, product ions 
appearing in the two are similar. They are also similar to those appearing in the high-energy CAD spectrum reported by Biemann et al. [15]. Following the previous report by Spengler et al. [30], prominent product ions in the PSD spectrum (Figure 1a) can be assigned to $a_{n}{ }^{\prime}(n=$ $1-3), a_{n}(n=5-8,10), a_{n}-\mathrm{NH}_{3}(n=1,5-8,10), b_{n}(n=2$, $3,5-10)$, and $b_{n}-\mathrm{NH}_{3}(n=2,3,5-8) ; a_{n}{ }^{\prime}$ ions appear at the same $m / z$ as the corresponding $a_{\mathrm{n}}$ ions in the PSD spectrum. From the H/D exchange study, Spengler et al. [30] showed that each $a_{n}{ }^{\prime}$ was either $a_{n}-\mathrm{NH}_{3}+\mathrm{OH}$ or a collection of internal ions. Some $y$ type ions also appear in PSD, $y_{10}$ being the most prominent. In the 193 nm PD spectrum, product ions with $\mathrm{m} / \mathrm{z}$ assignable to $a_{n}$ $(n=1-8,10)$ appear prominently. Since it is not clear at this point whether the first three in this series are genuine $a_{n}$ or $a_{n}{ }^{\prime}$, they will be denoted $a_{n} \cdot a_{n}-\mathrm{NH}_{3}(n=$ $1,2,5-8), b_{n}(n=2,3)$, and $b_{n}-\mathrm{NH}_{3}(n=2,3)$ also appear as in PSD. Among the product ions observed in PSD, $y$ type ions are virtually absent in PD. In contrast, $d$ type ions, which were absent in PSD, appear prominently in PD, viz. $d_{n}(n=3,5,6,10)$. Other prominent ions are $d_{7 \mathrm{Q}}$ and $a_{7}+1-\mathrm{R}_{\mathrm{Q}}$. According to Biemann et al. [15], $d_{7 \mathrm{Q}}$ is formed by the cleavage of the $\beta, \gamma$ bond of glutamine from the $a_{7}+1$ ion and $a_{7}+1-\mathrm{R}_{\mathrm{Q}}$ is formed from $(\mathrm{M}+\mathrm{H}-72)^{+}$, viz. loss of the glutamine side-chain from $(\mathrm{M}+\mathrm{H})^{+}$.

When the cell voltage is applied, each product ion peak in a PD-TOF spectrum usually split into more than one components as has been mentioned in the introductory section. The time-of-flight position of each component changes with the applied voltage. After the acquisition of a voltage-on spectrum, it has been our routine to linearly shift its $x$ (time)-scale such that the time-offlight of the precursor ion peak coincides with that in the voltage-off spectrum. Then, the time-of-flight position of the post-cell component of each product ion, which is due to its formation from the photoexcited precursor ion after the latter ion passes the cell, also coincides with the product ion position in the voltageoff spectrum [26]. This allows ready identification of the post-cell components, or post-cell peaks. The component of a product ion signal in the voltage-on spectrum that displays the largest deviation from the post-cell peak position is usually due to in-cell formation of the ion, while those in-between are due to its consecutive formation. Under the experimental condition used in PD of substance P ion, an in-cell peak is due to product ions formed within $110 \mathrm{~ns}$ after photoexcitation, while the corresponding post-cell peak is due to ions formed from the precursor ions during 0.18 to $4.9 \mu \mathrm{s}$ after photoexcitation. In Figure 2, the peak patterns for $a_{2}, b_{2}$, $a_{5}-\mathrm{NH}_{3}, d_{5}$, and $a_{7}$ in the $193 \mathrm{~nm}$ PD spectrum recorded with $3 \mathrm{kV}$ cell voltage are compared with those in the voltage-off spectrum. It can be seen that post-cell peaks are stronger than in-cell peaks for $a_{2}, b_{2}$, and $a_{5}-\mathrm{NH}_{3}$, while the opposite is the case for $a_{7}$. In the case of $d_{5}$, post-cell peak is nearly absent. Trends observed for other major peaks also belonged to one of the above three categories; dominant post-cell peaks for low mass $a_{n}(n=1-3), a_{n}-\mathrm{NH}_{3}, b_{n}$, and $b_{n}-\mathrm{NH}_{3}$, dominant in-cell peaks for high mass $a_{n}(n=4-8,10)$, and mostly in-cell peaks for $d_{n}$ and $d_{7 \mathrm{Q}}$.

Let us first consider a simple situation where all the dissociation channels for the precursor ion $\mathrm{M}^{+}$occur competitively and terminate in the first step, viz. consecutive reactions do not occur. Let us denote the rate constant for the production of each first-generation product ion $m_{j}^{+}(j=1-n)$, which will be called a primary product ion hereafter, as $k_{j}$. Then, the kinetics of formation of each $m_{j}^{+}$is governed by the total rate constant, which is the sum of $k_{j}$, not by the individual rate constant. The individual rate constant $k_{j}$ only determines the relative intensity of $m_{j}^{+}$. Then, all the primary product ions should show similar time-evolution pattern in the voltage-on spectrum. If consecutive reactions also occur, ions generated from the primary product ions, which will be called the secondary product ions, are not in competition with the primary product ions and can show different time-evolution patterns. Hence, participation of consecutive reactions can be an explanation for the appearance of three different time-evolution patterns in the voltage-on spectrum. However, there can be other explanations also. When one-step reactions of a precursor ion generate two different product ions, one rapidly and the other slowly, one often suspects the participation of nonstatistical processes. Two important assumptions in the statistical theory of mass spectra, RRKM-QET (quasiequilibrium theory) [31, 32], are rapid internal conversion to the ground electronic state and rapid intramolecular vibrational redistribution (IVR) in the ground electronic state. Whether the dissociation of an electronically excited peptide ion would satisfy the above two conditions has been a subject of controversy $[21,23,27,33,34]$. This question must be addressed before attempting to explain the appearance of three apparently different time-evolution patterns.

The electronic transition occurring at $193 \mathrm{~nm}$ is probably $\pi_{\mathrm{P}}{ }^{*} \leftarrow \pi_{\mathrm{P}}$ localized at peptide bonds [21, 35]. The first requirement for RRKM-QET would not be satisfied if the $\pi_{\mathrm{P}} \pi_{\mathrm{P}}{ }^{*}$ state thus accessed is dissociative. In contrast, the transition at $266 \mathrm{~nm}$ is localized at aromatic chromophores, viz. phenylalanine, tyrosine and tryptophan residues. Hence, it is unlikely that the excited-state accessed at $266 \mathrm{~nm}$ is also dissociative with respect to peptide bond cleavages. In our previous study [21], and also in Figure 1, we showed that the product ions formed by $193 \mathrm{~nm}$ PD were similar to those by $266 \mathrm{~nm}$ PD and suggested that the reactions observed occurred in the ground electronic state. As a further test, we recorded the voltage-on PD spectrum at $266 \mathrm{~nm}$. The peak patterns for the same ions as in Figure 2, viz. $a_{2}, b_{2}, a_{5}-\mathrm{NH}_{3}, d_{5}$, and $a_{7}$ are shown in Figure 3. Clearly, the splitting patterns at 266 and $193 \mathrm{~nm}$ are very similar and hence the first requirement for RRKMQET is probably met.

In one of our previous studies on $266 \mathrm{~nm}$ PD of peptide ions, it was observed that some product ions 

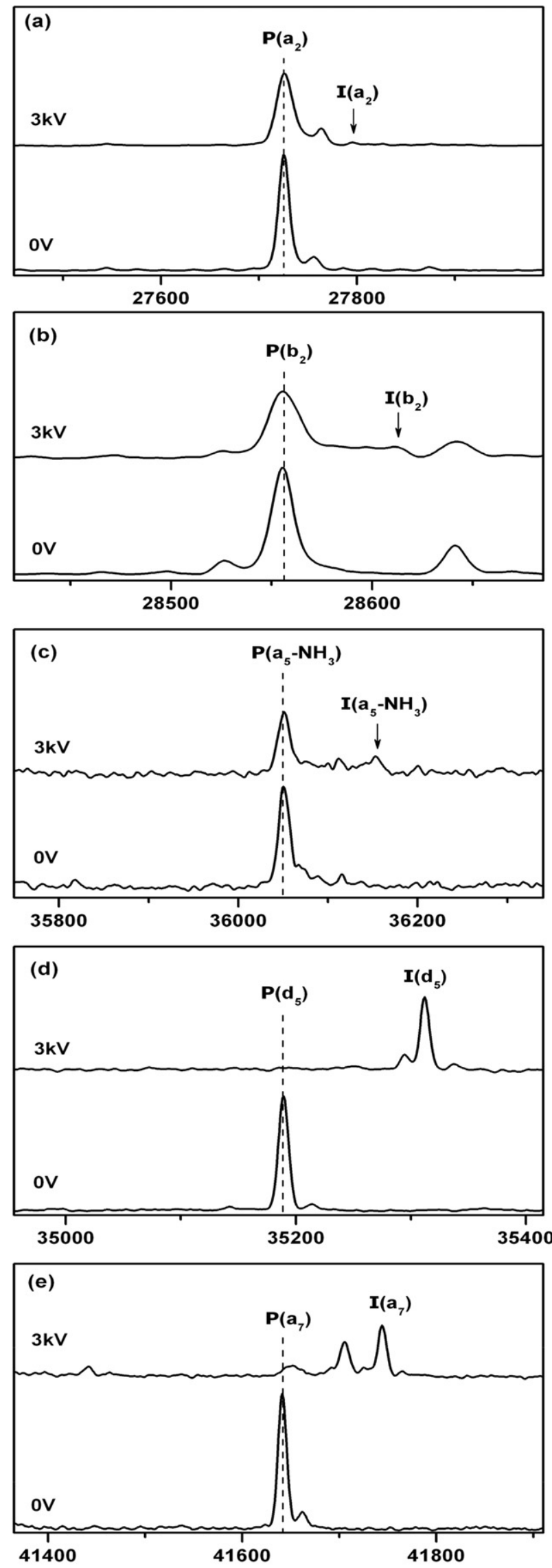

Time of flight, nsec formed by cleavage of peptide bonds immediately adjacent to an aromatic chromophore were particularly prominent [34]. It was suggested that the observation might be explained by postulating two types of dissociation, one occurring before complete IVR and the other after IVR. In this regard, it may be interesting to note that $d_{n}$ ions show mostly in-cell peaks in the voltage-on spectra. That is, formations of these ions are nearly completed within the cell unlike other product ions, suggesting their possible formation before complete IVR. Evidences against such a possibility can be found from the voltage-on $266 \mathrm{~nm}$ PD spectrum of substance $\mathrm{P}$ ion. Formation of $d_{6}$ is related to the cleavage of the peptide bond connected to the chromophore $(\mathrm{F})$ and is completed rapidly. In contrast, formations of $d_{3}, d_{5}$, and $d_{10}$, which are also rapidly completed, are related to the cleavage of bonds separated from the chromophore by one or more residues. We also observed post-cell $d$ peaks, even though weak, in $266 \mathrm{~nm}$ PD of some peptide ions, such as $d_{3}$ and $d_{6 a}$ from [RLLAPITAY $+\mathrm{H}]^{+}$. Such observations suggest that rapid or dominant formation of some product ions in UV-PD has nothing to do with incomplete IVR, viz. the second requirement for RRKM-QET is probably met. Based on the above arguments, it should be possible to explain, even though qualitatively, the appearance of three different time-evolution patterns within the statistical framework by taking into account the possibility of consecutive reactions.

Investigations on the dissociation of protonated peptides at low internal energy reported so far suggest that $b_{n}$ ions are primary product ions $[11,36-43] ; a_{n}$ ions may be formed via $\mathrm{CO}$ loss from $b_{n}[11,36,39,40]$. $a_{n}-\mathrm{NH}_{3}$ and $b_{n}-\mathrm{NH}_{3}$ are other secondary product ions appearing prominently at low internal energy, such as in the PSD spectrum shown in Figure 1a. As has been mentioned already, $a_{n}$ and $d_{n}$ are the most prominent product ions in the high-energy dissociation of protonated peptides with an arginine at the N-terminus. According to Biemann et al. [15], $d_{n}$ is formed from $a_{n}+$ 1 and $a_{n}$ from $a_{n}+1$ and $b_{n} \cdot a_{\mathrm{n}}+1$ ions are probably the primary product ions formed via the bond cleavage between $\mathrm{C}_{\alpha}$ and $\mathrm{CO}$, as noted by Reilly et al. [24]. Hence, it seems to be reasonable to postulate that the formations of $a_{n}+1$ and $b_{n}$ are the primary dissociation channels in direct competition in the present case and that other product ions are formed consecutively. Based on this picture, mostly post-cell formation of $a_{n}-\mathrm{NH}_{3}$ and $b_{n}-\mathrm{NH}_{3}$ can be readily explained by postulating slow $\mathrm{NH}_{3}$ loss from $a_{n}$ and $b_{n}$, respectively, or by postulating consecutive reactions via $\left[\mathrm{MH}-\mathrm{NH}_{3}\right]^{+}[11$, $43,44]$. Slow formation of low mass $a_{n}(n=1-3)$ can also

Figure 2. The peak patterns for (a) $a_{2}$, (b) $b_{2}$, (c) $a_{5}-\mathrm{NH}_{3}$, (d) $d_{5}$ and (e) $a_{7}$ in the $193 \mathrm{~nm}$ PD spectrum recorded with $3 \mathrm{kV}$ cell voltage. The patterns recorded without the cell voltage are shown at the bottom of each figure. Vertical dotted lines are drawn at the positions of post-cell $(\mathrm{P})$ peaks. In-cell peaks are marked I. 

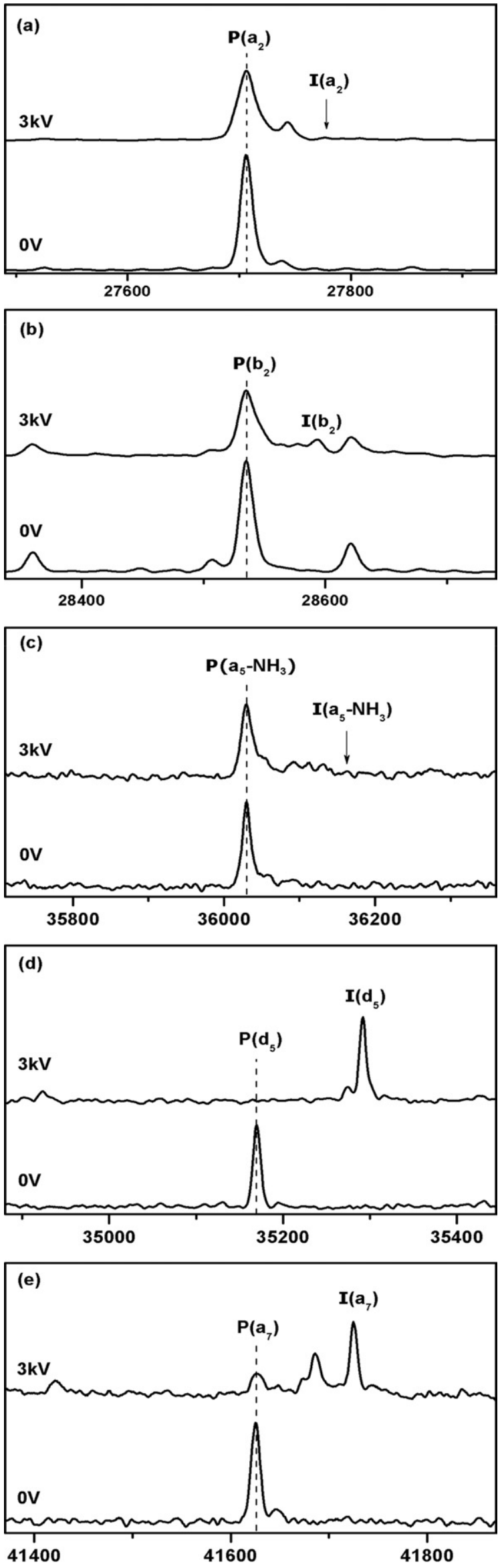

be explained by invoking consecutive reactions if these are $a_{n}{ }^{\prime}$, not genuine $a_{n}$, as found in PSD, even though further confirmation via $H / D$ exchange work is needed.

$b_{n}$ ions are formed via rearrangement reactions [3842] with small critical energies $\left(E_{0}\right)$ and small entropies of activation $\left(\Delta S^{\ddagger}\right)$. In contrast, both $\mathrm{E}_{0}$ and $\Delta \mathrm{S}^{\ddagger}$ are expected to be larger in the formation of $a_{n}+1$, which is a simple bond cleavage. As is well known in ion chemistry [4, 18], small $\mathrm{E}_{0}$ and $\Delta \mathrm{S}^{\ddagger}$ processes dominate at low internal energy, but give way to large $E_{0}$ and $\Delta S^{\ddagger}$ processes as the internal energy increases. Let us assume that $a_{n}+1$, which is a radical cation, dissociates rapidly to $a_{n}$ and $d_{n}$. Then, the striking difference in spectral pattern between PSD and UV-PD shown in Figure 1, that is, dominance of $b_{n}$ and its products, viz. $b_{n}-\mathrm{NH}_{3}, a_{n}$, and $a_{n}-\mathrm{NH}_{3}$ in PSD versus dominance of $a_{n}$ and $d_{n}$ in UV-PD, can be easily understood.

The main problem in the above explanation is the fact that two groups of competing primary channels, $b_{n}$ and $a_{n}+1$, seem to occur with noticeably different rate constants, mostly post-cell for the former reactions and mostly in-cell for the latter reactions. This is in apparent contradiction with the fact that the time-evolution patterns for all the competing channels are only governed by the total rate constant and should be the same. We can get out of this dilemma by recognizing that peptide ions generated by MALDI are not monoenergetic but have rather broad internal energy distributions (a sample calculation will be presented later). Accordingly, the internal energy distribution for photoexcited ions will also be broad. For a photoexcited precursor ion with sufficiently high internal energy, formation of $a_{n}+1$ will dominate. Such an ion will have a large total rate constant and dissociate rapidly inside the cell. $b_{n}$ may be formed at low-energy, even though weakly. Small total rate constant in this case would result in slow formation of $b_{n}$, mainly outside the cell.

The remaining difficulty concerns the fact that the time-evolution patterns of $d_{n}$ and high mass $a_{n}$ ions, which are thought to be formed competitively from $a_{n}+1$, are a little bit different. Experiments have consistently shown that the post-cell contribution for high mass $a_{n}$, even though very small, is more noticeable than that for $d_{n}$. Such a tiny difference may occur because $a_{n}+1$ is not monoenergetic. Also to be recalled is that $a_{n}+1$ is not the sole precursor for $a_{n}$, viz. $a_{n}$ can also be formed from $b_{n}$. To get more information on the nature of $a_{n}$ ions, both at low and high $\mathrm{m} / \mathrm{z}$, we attempted to differentiate these ions through H/D exchange and observe their time-evolution patterns.

At low internal energy, $a_{n}$ is thought to be formed from $b_{n}$ via CO loss $[39,40]$. The mechanism for the

Figure 3. The peak patterns for (a) $a_{2}$, (b) $b_{2}$, (c) $a_{5}-\mathrm{NH}_{3}$, (d) $d_{5}$ and (e) $a_{7}$ in the $266 \mathrm{~nm}$ PD spectrum recorded with $3 \mathrm{kV}$ cell voltage. The patterns recorded without the cell voltage are shown at the bottom of each figure. Vertical dotted lines are drawn at the positions of post-cell $(\mathrm{P})$ peaks. In-cell peaks are marked I. 

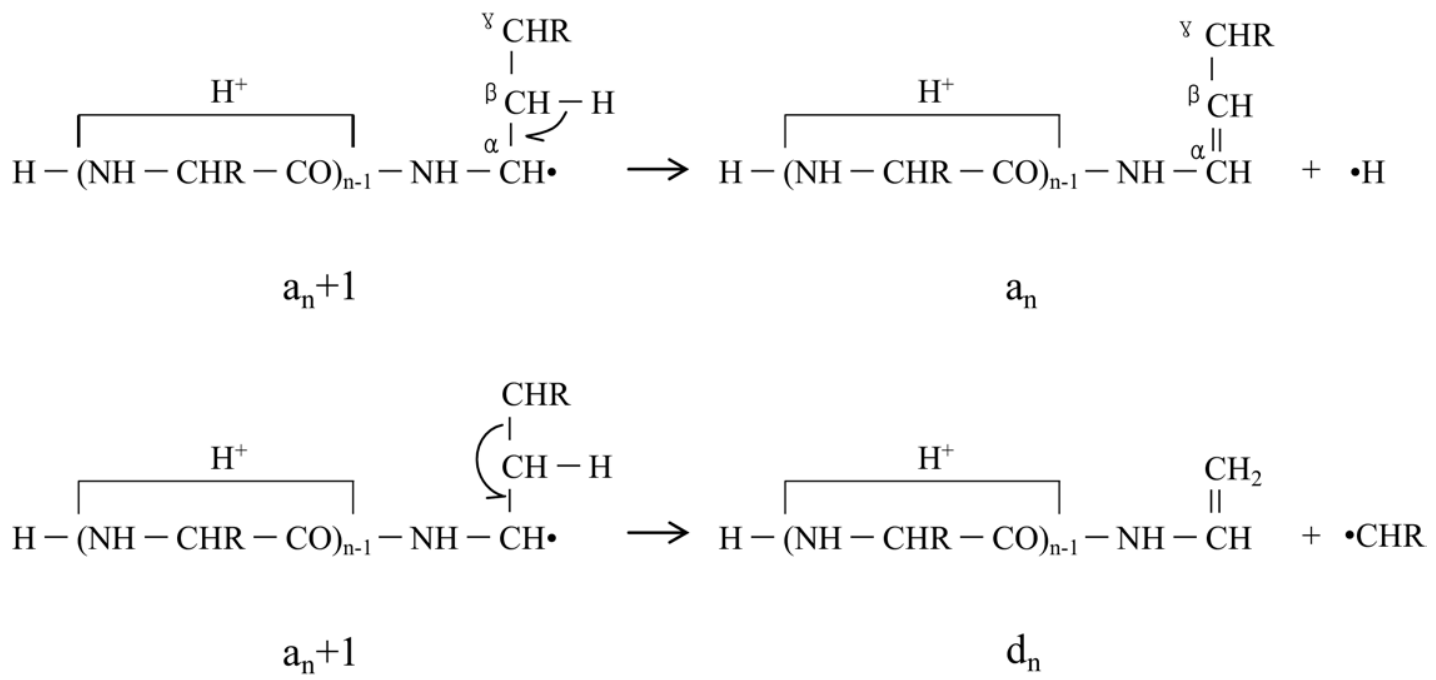

Scheme 2. Another structure of $a_{n}$ is shown in.

production of $b_{n}$ from a singly protonated peptide has been actively investigated. The general consensus [3842] reached is that the additional proton in the precursor ion migrates to the $\mathrm{N}$ atoms of amide bonds and the cleavage of these bonds with charge retention in the $\mathrm{N}$-terminal side results in $b_{n}$. In this model, the additional proton no longer remains in $b_{n}$, and hence neither in $a_{n}$. That is, when a deuteron is attached to substance $\mathrm{P}$, this will not be retained in $a_{n}$. At high internal energy, $a_{n}+1$ ions are the primary products. Requirement of charge retention in the $\mathrm{N}$-terminal side necessitates presence of the additional proton in the same side, probably at the arginine residue, which is the most basic. Biemann et al. [15] suggested that the radical site initiation at $\mathrm{C}_{\alpha}$ induces homolytic $\mathrm{C}_{\beta}-\mathrm{H}$ or $\mathrm{C}_{\beta}-\mathrm{C}_{\gamma}$ cleavage, resulting in $a_{n}$ or $d_{n}$ ions as shown in Scheme 2 .

The hydrogen atom at $C_{\beta}$ is not acidic and can not be deuterated by simple H/D exchange. Hence, two types of $a_{n}$ ions can be differentiated by H/D exchange; mass of $a_{n}$ formed via $b_{n}$, or $a_{n}^{l}$, is 1 Da lower than that via $a_{n}+$ 1 , or $a_{n}^{h}$. In the PSD study of deuterated substance P ion reported by Spengler et al. [30], all the high mass $a_{\mathrm{n}}$ ions were found to have the masses of $a_{n}^{l}$. In contrast, those of $a_{n}(n=1-3)$ were smaller by $2 \mathrm{Da}$, suggesting that they were not genuine $a_{n}$ ions. Reilly et al. [45] also used $\mathrm{H} / \mathrm{D}$ exchange in their structural study of $\alpha$-type ions formed by $157 \mathrm{~nm}$ PD in ion trap of peptide ions with an arginine at the $\mathrm{N}$-terminus. Two types of $a_{n}$ ions were observed, designated as $a_{n}+1-\mathrm{D}$ and $a_{n}+1-\mathrm{H}$. The latter ion is $a_{n}^{h}$ in our notation. $a_{n}+1-\mathrm{D}$, which appears at the same mass as $a_{n}^{l}$, was assumed to be formed via homolytic cleavage of the $\mathrm{N}-\mathrm{D}$ bond adjacent to $C_{\alpha}$. This mechanism was invoked to explain strong $a_{n}$ ions formed by cleavage at the C-terminal side of a glycine residue which does not have a $\beta$ hydrogen. It is to be noted that $a_{n}$ formed at the glycine site of substance P ion, viz. $a_{9}(m / z$ 1058.6), is very weak in our PD spectra. As mentioned above, generation of $d_{n}$ via homolytic $\mathrm{C}_{\beta}-\mathrm{C}_{\gamma}$ cleavage is another competitive dissociation channel for $a_{n}+1$. Favorable formation of $d_{n}$ at glutamic acid, glutamine, isoleucine, leucine, lysine, and valine sites were reported by Biemann et al. [15]. This is in agreement with the observation in the PD spectra obtained in this work (Figure 1).

Substance P has 22 exchangeable hydrogen atoms. Including a deuteron added in ion formation, the mass of substance $\mathrm{P}$ ion deuterated by $\mathrm{H} / \mathrm{D}$ exchange is larger by $23 \mathrm{Da}$ than that of the undeuterated ion. The molecular ion region of the deuterated substance $\mathrm{P}$ ion measured by MALDI-TOF in this work is shown in Figure 4. The peak at $\mathrm{m} / \mathrm{z} 1371.0$ is the A peak of the deuterated peptide ion. A peak due to incomplete deuteration also appears at $1 \mathrm{Da}$ below. Degree of

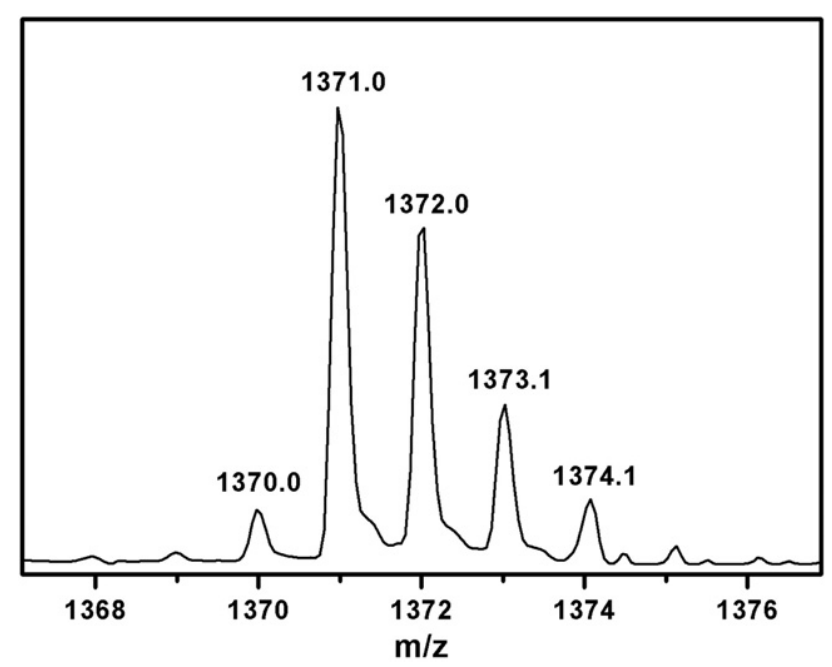

Figure 4. The molecular ion region of the MALDI-TOF spectrum of substance $\mathrm{P}$ deuterated by simple H/D exchange. The peak at $\mathrm{m} / \mathrm{z} 1371.0$ is the A peak of the deuterated peptide ion. 

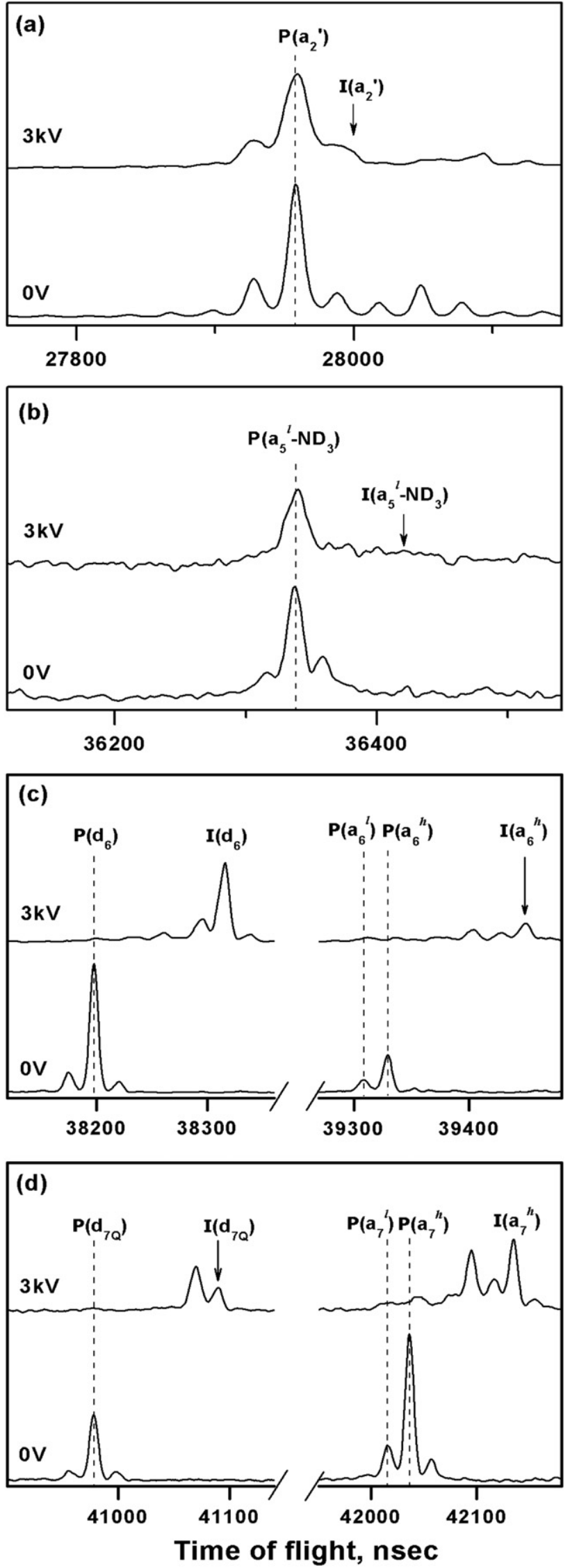

deuteration calculated from the isotopic pattern is around $99 \%$.

We recorded $193 \mathrm{~nm}$ PD spectrum for the A peak of the deuterated substance P ion. The full spectrum (not shown) is similar to that of the undeuterated ion once mass changes due to deuteration are taken into account. Some differences can be seen when the spectrum is expanded. Figure 5 shows the peak patterns of $a_{2}$, $a_{5}-\mathrm{ND}_{3},\left(d_{6}, a_{6}\right)$ pair, and $\left(d_{7 \mathrm{Q}}, a_{7}\right)$ pair recorded without and with the cell voltage. Mass of the main peak of $a_{2}$ (Figure 5a) is smaller than expected for $a_{n}^{l}$ by $2 \mathrm{Da}$, in agreement with Spengler et al. [30]. Then, dominance of the post-cell component for this $a_{n}{ }^{\prime}$ may be attributed to its consecutive formation. $a_{5}-\mathrm{NH}_{3}$ (Figure $5 b$ ) appears at the position expected for $a_{n}^{l}-\mathrm{ND}_{3}$, indicating that some $a_{n}$ ions are indeed formed from $b_{n} ; d_{6}$ (Figure $5 c$ ), and $d_{7 \mathrm{Q}}$ (Figure $5 \mathrm{~d}$ ) appear at the expected positions. Small additional peaks appear at $1 \mathrm{Da}$ below the main peaks in these cases. We do not know whether these additional peaks appear due to the experimental failure in monoisotopomeric selection or due to participation of other reactions. For $a_{6}$ (Figure $5 \mathrm{c}$ ) and $a_{7}$ (Figure 5d), peaks corresponding to $a_{n}^{l}$ (or $a_{n}+1-\mathrm{D}$ ) and $a_{n}^{h}$ are observed. The cell voltage-on patterns show that the post-cell contributions in $a_{6}^{h}$ and $a_{7}^{h}$ are very small, almost comparable to those in $d_{6}$ and $d_{7 \mathrm{Q}}$. The post-cell contributions in $a_{6}^{l}$ and $a_{7}^{l}$ (or $a_{n}+1-\mathrm{D}$ ) are also small, but are relatively larger than in $a_{n}^{h}$. The fact that the post-cell contributions in $a_{n}^{h}$ and $d_{n}$ are almost comparable suggests that the pathways leading to these product ions are competing effectively. Considering that the post-cell contribution for the peak at $1 \mathrm{Da}$ smaller mass is not large, it is unlikely that $a_{n}^{l}$ formed from $b_{n}$ is the sole contributor to this peak. Sole contribution from $a_{n}+1-\mathrm{D}$ is unlikely either because its post-cell contribution is not as important as for $a_{n}^{h}$. These peaks may be combinations of $a_{n}^{l}$ and $a_{n}+1-\mathrm{D}$. The important thing is that separating these peaks from $a_{n}^{h}$ results in rather similar time-evolution patterns for $a_{n}^{h}$ and $d_{n}$. We also performed $193 \mathrm{~nm}$ PD for a few other deuterated peptide ions. Peak patterns of $d_{4 \mathrm{a}}$ and $a_{4}$ from [RVYIPF + $\mathrm{D}^{+}$are shown in Figure 6. Here again, the post-cell contribution in $a_{n}^{h}$ is very small while that at 1 Da lower is much larger.

We have suggested that dissociation of an electronically excited peptide ion with an arginine at the $\mathrm{N}$ terminus, either in UV-PD or in high-energy CAD, occurs statistically in the ground state. Two sets of channels, simple homolytic cleavages to $a_{n}+1$ (high $\mathrm{E}_{0}$ and high $\Delta \mathrm{S}^{\ddagger}$ ) and rearrangement reactions to $b_{n}$ (low $\mathrm{E}_{0}$ and low $\Delta S^{\ddagger}$ ), were assumed to be in competition. A

Figure 5. The peak patterns for (a) $a_{2}{ }^{\prime}$, (b) $a_{5}-\mathrm{ND}_{3}$, (c) $\left(d_{6}, a_{6}\right)$ pair, and $(\mathbf{d})\left(d_{7 \mathrm{O}}, a_{7}\right)$ pair in the $193 \mathrm{~nm}$ PD spectrum of deuterated substance P recorded with $3 \mathrm{kV}$ cell voltage. The patterns recorded without the cell voltage are shown at the bottom of each figure. Vertical dotted lines are drawn at the positions of post-cell (P) peaks. In-cell peaks are marked I. 


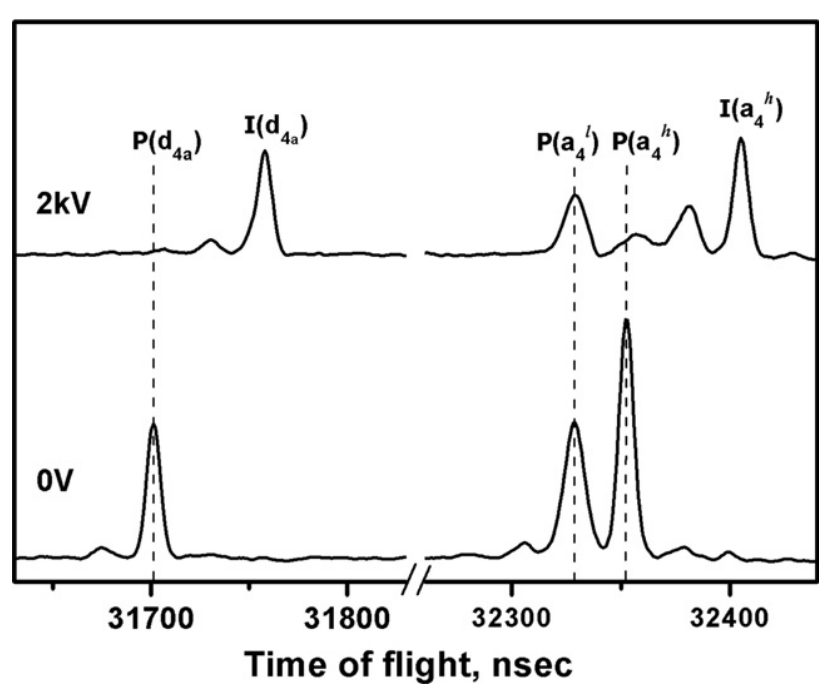

Figure 6. The peak patterns for $d_{4 \mathrm{a}}$ and $a_{4}$ in the $193 \mathrm{~nm}$ PD spectrum of deuterated [RVYIPF $+\mathrm{D}]^{+}$recorded with $2 \mathrm{kV}$ cell voltage. The patterns recorded without the cell voltage are shown at the bottom of the figure. Vertical dotted lines are drawn at the positions of post-cell $(\mathrm{P})$ peaks. In-cell peaks are marked I.

broad internal energy distribution for a photoexcited peptide ion originating from the thermal energy acquired at the time of ion formation (MALDI) was invoked to explain the different time-evolution patterns for these two channels. For a rigorous test of this model, accurate data on internal energy distribution and rate constants are needed, which are not available at the moment. In the theoretical estimation of the rate-energy $(\mathrm{k}(\mathrm{E}))$ data, particularly troublesome is the $b_{n}$ channel, which is thought to occur along a reaction pathway consisting of several isomerization/tautomerization steps [38, 46]. It is uncertain how the rate constant in each step would influence the overall rate constant. Since the main purpose of the present effort was just to demonstrate the feasibility of the statistical picture in explaining the experimental observations, a drastically simple approach was taken. That is, the statistical dissociation rate constants to $a_{n}+1$ and $b_{n}$ for substance $P$ ions were calculated by RRKM-QET as if each of them occurred via a hypothetical one-step reaction. The software reported previously [5-7] was used in the calculation. The software also calculates the internal energy distribution for a precursor ion at a specified temperature. Since the software in its present form can handle regular peptides and peptide ions only, not amide derivatives such as substance $\mathrm{P}$, a minor adjustment would be needed. This has not been made in this work because the outcomes are expected to be nearly the same regardless of the adjustment. As the reaction coordinate, a mode at $1150 \mathrm{~cm}^{-1}$, which is a default in the software, was chosen. Use of other modes did not noticeably affect the rate-energy $(\mathrm{k}(\mathrm{E}))$ curve.

It is usual to treat peptide ions generated by MALDI as if they were in thermal equilibrium [47, 48]. To calculate the internal energy distribution of a peptide ion with this model, its effective temperature is needed. It is a parameter that can not be easily measured. Also, it may change significantly depending on the MALDI condition used. It can be anywhere between 500 and $1000 \mathrm{~K}$, or even higher [47, 49]. The parameters needed to calculate the rate-energy curve for each channel are $\mathrm{E}_{0}$ and $\Delta \mathrm{S}^{\ddagger}$. None of these parameters are accurately known for $a_{n}+1$ and $b_{n}$ channels. From quantum chemical studies, Paizs and Suhai $[38,39]$ suggested $E_{0}$ of 0.65 to $1.1 \mathrm{eV}$ for $b-y$ dissociation channels for peptide ions without an arginine residue. No theoretical study has been reported for $b-y$ channels for peptide ions with this residue. It is thought that a statistical description of this case would require an $\mathrm{E}_{0}$ value larger than the arginine-free case. In an $a_{n}+1$ channel, a C-C bond is broken. Its $\mathrm{E}_{0}$ may be smaller than the typical $\mathrm{C}-\mathrm{C}$ bond energy of $345 \mathrm{~kJ} \mathrm{~mol}^{-1}(3.6 \mathrm{eV})$ due to stabilization of the radical sites in the ionic and neutral products. In dissociation of small molecules, $\Delta S^{\ddagger}$ for a reaction occurring via a tight transition-state (rearrangement) is near $0 \mathrm{eu}\left(1 \mathrm{eu}=4.184 \mathrm{~J} \mathrm{~K}^{-1} \mathrm{~mol}^{-1}\right)$ while that via a loose transition-state (simple bond cleavage) is near 15 eu $[31,33] . \Delta S^{\ddagger}$ for $a_{n}+1$ channels may be even larger considering the complexity of peptide ions. Keeping in mind the main purpose of the present effort, we took freedom in choosing values for the above parameters.

To calculate the $\mathrm{k}(\mathrm{E})$ curve for a $b_{n}$ channel in substance $P$ ion, we used $\mathrm{E}_{0}=1.4 \mathrm{eV}$ and $\Delta \mathrm{S}^{\ddagger}=0.0 \mathrm{eu}$. Then, $\mathrm{k}(\mathrm{E})$ thus obtained for a single $b_{n}$ channel was multiplied by 10 to mimic participation of many such channels. Similar calculation was done for $a_{n}+1$ channels using $\mathrm{E}_{0}=2.0 \mathrm{eV}$ and $\Delta \mathrm{S}^{\ddagger}=20 \mathrm{eu}$. The results are shown in Figure 7. Change in the dominant channel, from $b_{n}$ at low-energy to $a_{n}+1$ at high-energy, can be seen in the figure. The rate constant estimated from the flight time of a photoexcited ion inside the cell is marked in the figure. This is approximately the minimum rate constant needed for in-cell dissociation. The rate constant range for the appearance of PSD is also marked in the figure; $700 \mathrm{~K}$ was taken as the effective temperature of substance $\mathrm{P}$ ion generated by MALDI. Its internal energy distributions before and after the absorption of one $193 \mathrm{~nm}$ photon are also shown in Figure 7. It can be seen from the figure that $b_{n}$ channels dominate in PSD, which is driven by the internal energy acquired in MALDI. With the addition of one $193 \mathrm{~nm}$ photon, rapid in-cell dissociation can occur mostly via $a_{n}+1$ channels, even though slower post-cell dissociation via $b_{n}$ channels is also possible for photoexcited ions with internal energy falling in the lower part of the distribution. The overall situation is in agreement with the statistical interpretation presented in this paper. We do not claim that $k(E)$ and energy distribution curves shown in Figure 7, the magnitude of each parameter used in particular, are correct descriptions of the situation. By using other sets of parameters, we could obtain results that were compatible with observations. The point here is that it is possible to explain the different dissociation patterns for a peptide ion with an arginine 


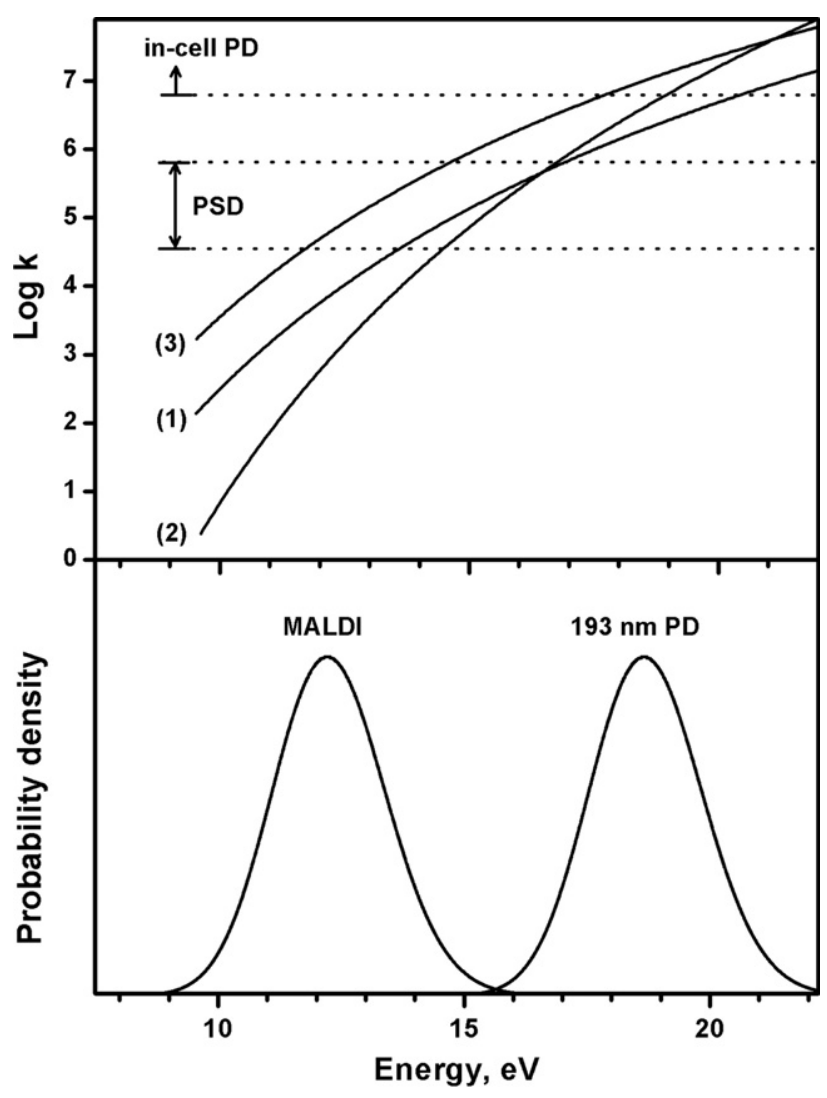

Figure 7. $k(E)$ curves for dissociation of substance $\mathrm{P}$ ions calculated with (1) $\mathrm{E}_{0}=1.4 \mathrm{eV}, \Delta \mathrm{S}^{\ddagger}=0.0 \mathrm{eu},(2) \mathrm{E}_{0}=2.0 \mathrm{eV}, \Delta \mathrm{S}^{\ddagger}=20$ eu, and (3) $\mathrm{E}_{0}=1.28 \mathrm{eV}, \Delta \mathrm{S}^{\ddagger}=0.0$ eu. (1) and (2) are model calculations for $b_{n}$ and $a_{n}+1$ channels, respectively; (3) is a fictitious model for $b_{n}$ channels in the absence of arginine. The internal energy distributions for the same ion before (marked as MALDI) and after (marked as $193 \mathrm{~nm}$ PD) absorption of one 193 $\mathrm{nm}$ photon are shown at the bottom; $700 \mathrm{~K}$ was taken as the effective temperature of the ion generated by MALDI.

at the N-terminus, PSD versus UV-PD or low versus high-energy CAD, within the statistical framework.

We have mentioned earlier that characteristic patterns in UV-PD, and also in high-energy CAD, were observed for peptide ions with an arginine residue. For peptide ions without this residue $[11,39], b_{n}$ and $y_{n}$ ions dominated just as in PSD and low-energy CAD. We were curious to know whether such an insensitivity of the spectral pattern could also be explained within the statistical framework. Use of an $\mathrm{E}_{0}$ value in the theoretically predicted range 0.65 to $1.1 \mathrm{eV}$ [39] was found to be inadequate because $\mathrm{k}(\mathrm{E})$ thus obtained predicted complete disappearance of the precursor ion by PSD. To avoid this, we calculated $\mathrm{k}(\mathrm{E})$ using $\mathrm{E}_{0}=1.28 \mathrm{eV}$ and $\Delta S^{\ddagger}=0.0 \mathrm{eu}$. The result is also shown in Figure 7. In this case, $b_{n}$ channels dominate almost over the entire energy range covered, in agreement with experimental observations. This suggests that the striking characteristics in high-energy CAD and UV-PD-dominance of $a_{\mathrm{n}}$ and $d_{\mathrm{n}}$ - of peptide ions with an arginine at the $\mathrm{N}$-terminus are not due to the peculiarity of the excitation processes themselves, but due to quenching of the dominant low-energy channels caused by the presence of arginine. It is also to be noted that a slight difference in $\mathrm{E}_{0}, 1.40$ versus $1.28 \mathrm{eV}$ in the present calculations, can lead to the dramatic difference in spectral pattern between peptide ions with and without an arginine, even though the internal energies of activated peptide ions are as large as $20 \mathrm{eV}$.

\section{Conclusions}

Substantially different time-evolution patterns for different product ion types were observed in UV-PD experiments for singly protonated peptides with an arginine residue at the $\mathrm{N}$-terminus using a voltagefloated cell, as if nonstatistical processes had participated. Additional experiments indicated, however, that dissociations occurred statistically in the ground electronic states. The apparent conflict could be reconciled by invoking that some product ions were formed via consecutive reactions and that peptide ions generated by MALDI had a rather broad internal energy distribution. It was suggested that rearrangement reactions to $b_{n}$ and homolytic cleavages to $a_{n}+1$ were the first step dissociation channels which were in competition in the energy range covered. Feasibility of this model was demonstrated through rough internal energy and RRKM calculations. The results were compatible with the observations that $b_{n}$ channels were dominant in PSD and low-energy CAD while $a_{n}+1$ channels became important in UV-PD and high-energy CAD. In the case of peptide ions without an arginine residue, a sample calculation predicted dominance of $b_{n}$ channels over the entire energy range covered, again in agreement with observations. Specifically, the striking characteristics in high-energy CAD and UV-PD-dominance of $a_{\mathrm{n}}$ and $d_{\mathrm{n}}$ - of peptide ions with an arginine at the N-terminus were not due to the peculiarity of the excitation processes themselves, but due to quenching of the dominant low-energy channels caused by the presence of arginine.

Present statistical calculations were made under the dramatically simplistic assumption of one-step reaction for each channel. They also suffer from the lack of knowledge on the internal energy distribution for peptide ions and dynamical parameters governing their dissociation. We hope that the reliability of the statistical calculation will improve with further investigations, both experimental and theoretical. Then, one may be able to check the participation of an alternative nonstatistical mechanism by comparing with statistically expected results.

\section{Acknowledgments}

The authors gratefully acknowledge financial support for this work by the Korea Research Foundation, Republic of Korea, and by the Biosignal Analysis Technology Innovation program (M10645010002-06N4501-00,210) of the Ministry of Science and 
Technology, Republic of Korea. SHY and YJC thank the Ministry of Education, Republic of Korea, for Brain Korea 21 Fellowship.

\section{References}

1. Mann, M.; Hendrickson, R. C.; Pandey, A. Analysis of Proteins and Proteomes by Mass Spectrometry. Annu. Rev. Biochem. 2001, 70, 437473.

2. Kinter, M.; Sherman, N. E. Protein Sequencing and Identification Using Tandem Mass Spectrometry; John Wiley: New York, 2000; pp. 64-116, pp. $238-268$.

3. Hernandez, P.; Müller, M.; Appel, R. D. Automated Protein Identification by Tandem Mass Spectrometry: Issues and Strategies. Mass Spectrom. Rev. 2006, 25, 235-254.

4. Bowers, M. T., Ed. In Gas Phase Ion Chemistry, Vol. III, Ions and Light, Eds.; Academic Press: Orlando, FL, 1984

5. Moon, J. H.; Oh, J. Y.; Kim, M. S. A Systematic and Efficient Method to Estimate the Vibrational Frequencies of Linear Peptide and Protein Ions with Any Amino Acid Sequence for the Calculation of Rice-Ramsperger-Kassel-Marcus Rate Constant. J. Am. Soc. Mass Spectrom. 2006, $17,1749-1757$.

6. Sun, M.; Moon, J. H.; Kim, M. S. Improved Whitten-Rabinovitch Approximation for the Rice-Ramsperger-Kassel-Marcus Calculation of Unimolecular Reaction Rate Constant for Proteins. J. Phys. Chem. B 2007, 111, 2747-2751.

7. Moon, J. H.; Sun, M.; Kim, M. S. Efficient and Reliable Calculation of Rice-Ramsperger-Kassel-Marcus Unimolecular Reaction Rate Constants for Biopolymers: Modification of Beyer-Swinehart Algorithm for Degenerate Vibrations. J. Am. Soc. Mass Spectrom. 2007, 18, 1063-1069.

8. McLafferty, F. W.; Ed. Tandem Mass Spectrometry, Wiley-Interscience: New York, 1983; pp 125-174.

9. Cooks, R. G. 1978; Collision-Induced Dissociation of Polyatomic Ions. Cooks, R. G., Ed.; In Collision Spectroscopy; pp 375-450.Plenum: New York,

10. Martin, S. A.; Biemann, K. 1990, Sequencing of Peptides by Tandem Mass Spectrometry and High-Energy Collision-Induced Dissociation. McCloskey, J. A., Ed.; In Methods in Enzymology, Vol. CXCIII, Mass Spectrometry; pp 455-479.Academic Press: New York,

11. Medzihradszky, K. F. 2005, Peptide Sequence Analysis. Burlingame, A. L., Ed.; In Methods in Enzymology, Vol. CDII, Biological Mass Spectrometry; pp 209-244.Academic Press: San Diego,

12. Laskin, J.; Futrell, J. H.; Collisional Activation of Peptide Ions in FT-ICR Mass Spectrometry. Mass Spectrom. Rev. 2003, 22, 158-181.

13. Wysocki, V. H.; Tsaprailis, G.; Smith, L. L. Breci, L. A. Mobile and Localized Protons: A Framework for Understanding Peptide Dissociation. J. Mass Spectrom. 2000, 35, 1399-1406.

14. Summerfield, S. G.; Whiting, A.; Gaskell, S. J. Intra-Ionic Interactions in Electrosprayed Peptide Ions. Int. J. Mass Spectrom. Ion Processes 1997, $162,149-161$.

15. Johnson, R. S.; Martin, S. A.; Biemann, K. Collision-Induced Fragmentation of $(\mathrm{M}+\mathrm{H})^{+}$Ions of Peptides. Side Chain Specific Sequence Ions. Int. J. Mass Spectrom. Ion Processes 1988, 86, 137-154.

16. Little, D. P.; Sper, J. P.; Senko, M. W.; O'Connor, P. B.; McLafferty, F. W. Infrared Multiphoton Dissociation of Large Multiply Charged Ions for Biomolecule Sequencing. Anal. Chem. 1994, 66, 2809-2815.

17. Laskin, J.; Futrell, J. H. Activation of Large Ions in FT-ICR Mass Spectrometry. Mass Spectrom. Rev. 2005, 24, 135-167.

18. Bowers, M. T., Ed.; Gas Phase Ion Chemistry, Vol. II, Eds.; Academic Press: New York, 1979; pp 181-220.

19. Dunbar, R. C. Photodissociation of Trapped Ions. Int. J. Mass Spectrom. 2000, 200, 571-589.

20. Moon, J. H.; Yoon, S. H.; Kim, M. S. Photodissociation of Singly Protonated Peptides at $193 \mathrm{~nm}$ Investigated with Tandem Time-ofFlight Mass Spectrometry. Rapid Commun. Mass Spectrom. 2005, 19, 3248-3252.

21. Choi, K. M.; Yoon, S. H.; Sun, M.; Oh, J. Y.; Moon, J. H.; Kim, M. S. Characteristics of Photodissociation at $193 \mathrm{~nm}$ of Singly Protonated Peptides Generated by Matrix-Assisted Laser Desorption Ionization (MALDI). J. Am. Soc. Mass Spectrom. 2006, 17, 1643-1653.

22. Barbacci, D. C.; Russell, D. H. Sequence and Side-Chain Specific Photofragment $(193 \mathrm{~nm})$ Ions from Protonated Substance P by MatrixAssisted Laser Desorption Ionization Time-of-Flight Mass Spectrometry. J. Am. Soc. Mass Spectrom. 1999, 10, 1038-1040.

23. Thompson, M. S.; Cui, W.; Reilly, J. P.Fragmentation of Singly Charged Peptide Ions by Photodissociation at $\lambda=157 \mathrm{~nm}$.Angew. Chem. Int. Ed. 200443 4791-4794.

24. Cui, W.; Thompson, M. S.; Reilly, J. P. Pathways of Peptide Ion Fragmentation Induced by Vacuum Ultraviolet Light. J. Am. Soc. Mass Spectrom. 2005, 16, 1384-1398.
25. Moon, J. H.; Shin, Y. S.; Cha, H. J.; Kim, M. S. Photodissociation at 193 $\mathrm{nm}$ of Some Singly Protonated Peptides and Proteins with $\mathrm{m} / \mathrm{z} 2000$ 9000 Using a Tandem Time-of-Flight Mass Spectrometer Equipped with a Second Source for Delayed Extraction/Post-Acceleration of Product Ions. Rapid Commun. Mass Spectrom. 2007, 21, 359-368.

26. Yoon, S. H.; Kim, M. S. Development of a Time-Resolved Method for Photodissociation Mechanistic Study of Protonated Peptides: Use of a Voltage-Floated Cell in a Tandem Time-of-Flight Mass Spectrometer. J. Am. Soc. Mass Spectrom. 2007, 18, 1729-1739.

27. Morgan, J. W.; Russell, D. H. Comparative Studies of 193-nm Photodissociation and TOF-TOFMS Analysis of Bradykinin Analogues: The Effects of Charge Site and Fragmentation Timescales. J. Am. Soc. Mass Spectrom. 2006, 17, 721-729.

28. Yoon, S. H.; Moon, J. H.; Choi, K. M.; Kim. M. S. A Deflection System to Reduce the Interference from Post-Source Decay Product Ions in Photodissociation Tandem Time-of-Flight Mass Spectrometry. Rapid Commun. Mass Spectrom. 2006, 20, 2201-2208.

29. Moon, J. H.; Yoon, S. H.; Kim. M. S. Enhancement of Matrix-Assisted Laser Desorption/Ionization Photodissociation Tandem Time-of-Flight Mass Spectra: Spectral Reduction and Cleanup of Isotopomeric Contamination. Rapid Commun. Mass Spectrom. 2005, 19, 2481-2487.

30. Spengler, B.; Lützenkirchen, F.; Kafumann, R. On-Target Deuteration for Peptide Sequencing by Laser Mass Spectrometry. Org. Mass Spectrom. 1993, 28, 1482-1490.

31. Baer, T.; Hase, W. L. Unimolecular Reaction Dynamics: Theory and Experiments, Eds.; Oxford University Press: New York, 1996; pp 171-415.

32. Holbrook, K. A.; Pilling, M. J.; Robertson, S. H. Unimolecular Reactions; Wiley: Chichester, 1996; pp 39-78

33. Hu, Y.; Hadas, B.; Davidovitz, M.; Balta, B.; Lifshitz, C. Does IVR Take Place Prior to Peptide Ion Dissociation? J. Phys. Chem. A 2003, 107, 6507-6514.

34. Oh, J. Y.; Moon, J. H.; Kim, M. S. Sequence- and Site-Specific Photodissociation at $266 \mathrm{~nm}$ of Protonated Synthetic Polypeptides Containing a Tryptophanyl Residue. Rapid Commun. Mass Spectrom. 2004, 18, $2706-$ 2712 .

35. Robin, M. B. Higher Excited States of Polyatomic Molecules, Vol. II, Eds. Academic Press: New York, 1974; pp 122-155.

36. Papayannopoulos, I. A. The Interpretation of Collision-Induced Dissociation Tandem Mass Spectra of Peptides. Mass Spectrom. Rev. 1995, 47, 49-73.

37. Kaufmann, R.; Spengler, K. B. Sequencing of Peptides in a Time-ofFlight Mass Spectrometer: Evaluation of Post-Source Decay Following Matrix-Assisted Laser Desorption Ionization. Int. J. Mass Spectrom. Ion Processes 1994, 131, 355-385.

38. Paizs, B.; Suhai, S. Combined Quantum Chemical and RRKM Modeling of the Main Fragmentation Pathways of Protonated GGG. II. Formation $\mathrm{b}_{2}, \mathrm{y}_{1}$, and $\mathrm{y}_{2}$ Ions. Rapid Commun. Mass Spectrom. 2002, 16, 375-389.

39. Paizs, B.; Suhai, S. Fragmentation Pathways of Protonated Peptides. Mass Spectrom. Rev. 2005, 24, 508-548.

40. El Aribi, H.; Rodriquez, C. F.; Almeida, D. R. P.; Ling, Y.; Mak, W. W.-N.; Hopkinson, A. C.; Siu, K. W. M. Elucidation of Fragmentation Mechanisms of Protonated Peptide Ions and Their Products: A Case Study on Glycylglycylglycine Using Density Functional Theory and Threshold Collision-Induced Dissociation. J. Am. Chem. Soc. 2003, 125 9229-9236.

41. Polce, M. J.; Ren, D.; Wesdemiotis, C. Dissociation of the Peptide Bond in Protonated Peptides. J. Mass Spectrom. 2000, 35, 1391-1398.

42. Schlosser, A.; Lehmann, W. D. Five-Membered Ring Formation in Unimolecular Reaction of Peptides: A Key Structural Element Controlling Low-Energy Collision-Induced Dissociation of Peptides. J. Mass Chem. 2000, 35, 1382-1390.

43. Laskin, J.; Bailey, T. H.; Futrell, J. H. Mechanisms of Peptide Fragmentation from Time- and Energy-Resolved Surface-Induced Dissociation Studies: Dissociation of Angiotensin Analogs. Int. J. Mass Spectrom. 2006 249/250, 462-472

44. Subra, G.; Aubagnac, J. L.; Martinez, J.; Enjalbal, C. Tandem Mass Spectrometry of Amidated Peptides. J. Mass Spectrom. 2006, 41, 1470 1483 .

45. Zhang, L.; Cui, W.; Thompson, M. S.; Reilly, J. P. Structures of $\alpha$-Type Ions Formed in the $157 \mathrm{~nm}$ Photodissociation of Singly-Charged Peptide Ions. J. Am. Soc. Mass Spectrom. 2006, 17, 1315-1321.

46. Rodriquez, C. F.; Cunje, A.; Shoeib, T.; Chu, I. K.; Hopkinson, A. C.; Siu, K. W. M. Proton Migration and Tautomerism in Protonated Triglycine. J. Am. Chem. Soc. 2001, 123, 3006-3012.

47. Zenobi, R.; Knochenmuss, R. Ion Formation in MALDI Mass Spectrometry. Mass Spectrom. Rev. 1998, 17, 337-366.

48. Dreisewerd, K. The Desorption Process in MALDI. Chem. Rev. 2003, 103, 395-425.

49. Koubenakis, A.; Frankevich, V.; Zhang, J.; Zenobi, R. Time-Resolved Surface Temperature Measurement of MALDI Matrices under Pulsed UV Laser Irradiation. J. Phys. Chem. A 2004, 108, 2405-2410. 